\title{
Development of micromechanics for micro-autonomous systems (ARL- MAST CTA Program)
}

J. S. Humbert, I. Chopra, R. S. Fearing, R. J. Full, R. J. Wood, et al.

J. S. Humbert, I. Chopra, R. S. Fearing, R. J. Full, R. J. Wood, M. H. Dickinson, "Development of micromechanics for micro-autonomous systems (ARL-MAST CTA Program)," Proc. SPIE 7318, Micro- and Nanotechnology Sensors, Systems, and Applications, 73180L (11 May 2009); doi: $10.1117 / 12.820881$

Event: SPIE Defense, Security, and Sensing, 2009, Orlando, Florida, United States 


\title{
Development of Micromechanics for Micro Autonomous Systems (ARL-MAST CTA Program)
}

\author{
J. S. Humbert ${ }^{a}$, I. Chopra ${ }^{a}$, R. S. Fearing ${ }^{b}$, R. J. Full ${ }^{b}$, R. J. Wood ${ }^{c}$, and M. H. Dickinson ${ }^{d}$ \\ ${ }^{a}$ University of Maryland, College Park, MD \\ ${ }^{b}$ University of California, Berkeley, CA \\ ${ }^{c}$ Harvard University, Cambridge, MA \\ ${ }^{d}$ California Institute of Technology, Pasadena, CA
}

\begin{abstract}
We envision situational awareness developed through warfighters deployment of a system of diverse mobile, communicating platforms that cooperate to provide full coverage of interior and exterior spaces. The goal of the ARL-MAST Center on Microsystem Mechanics is to perform the fundamental research that will enable flying and ambulating platforms to achieve the required mobility for the proposed missions and environments. In this paper the fundamental issues and challenges associated with achieving this goal will be discussed.
\end{abstract}

Keywords: Autonomous mobility, sensing, control, and navigation

\section{INTRODUCTION}

The goal of the Center on Microsystem Mechanics is to create micromechanical systems to achieve penetration and reconnaissance for missions of interest to the Army, focusing on fundamental research related to platform mobility within complex, unknown environments. In the future, we envision that a squad or individual warfighter will deploy a system of cooperating and communicating mobile microsystems. Large flyers could quickly cover large distances, provide high sensing, computation and communication ability, and opportunistically deploy smaller fixed and mobile platforms. Smaller fliers penetrate as far as possible, perch, collect data and transmit, but also search for passages only possible for small crawlers/jumpers. Crawlers/jumpers can operate in high gust conditions, have long duration, and penetrate openings too small for the smallest flyers. The crawler/flyer combination provides hybrid locomotion, opportunistically switching from ambulation to flight when terrain is impassable (e.g. cave walls coated with a viscous fluid) and switching from flight to crawling in high gust conditions or confined spaces. Figure 1 broadly shows the envisioned platforms and a summary of their capabilities. Key characteristics that are ubiquitous to any agent morphology include:

- Multi-modal locomotion: indoor flight and/or all-terrain ambulation. Agents will need to quickly and efficiently traverse the exterior and interior of the cave. For ambulation, this translates to the ability to move over obstacles, debris, varying surfaces, and potentially up walls. For flight, this translates to the ability to hover so that an agent can effectively move in a constricted environment.

- Perch and stare: the overall goal is to collect pertinent information about the contents of the cave. This requires two things from an individual agent: the ability to hold position and sufficient payload to carry sensors that are appropriate for the mission.

- Stealthy operation: centimeter-scale agents operating in a cluttered environment (whether flying or walking) will naturally exhibit stealth-by-mimicry. To expand upon this, it is important that the agents are quiet (acoustically, RF, optically, etc).

- Robust operation: this implies that the agents are individually robust to potential collisions with obstacles in the environment. This also implies that groups of agents operating as a swarm are robust to individual agent failures and changes in the environment, mission, etc.

Send correspondence to humbert@umd.edu

Micro- and Nanotechnology Sensors, Systems, and Applications, edited by Thomas George, M. Saif Islam, Achyut K. Dutta, Proc. of SPIE Vol. 7318, 73180L · C 2009 SPIE · CCC code: 0277-786X/09/\$18 · doi: 10.1117/12.820881 


\section{Distributed Mobility through Multiple Scales}

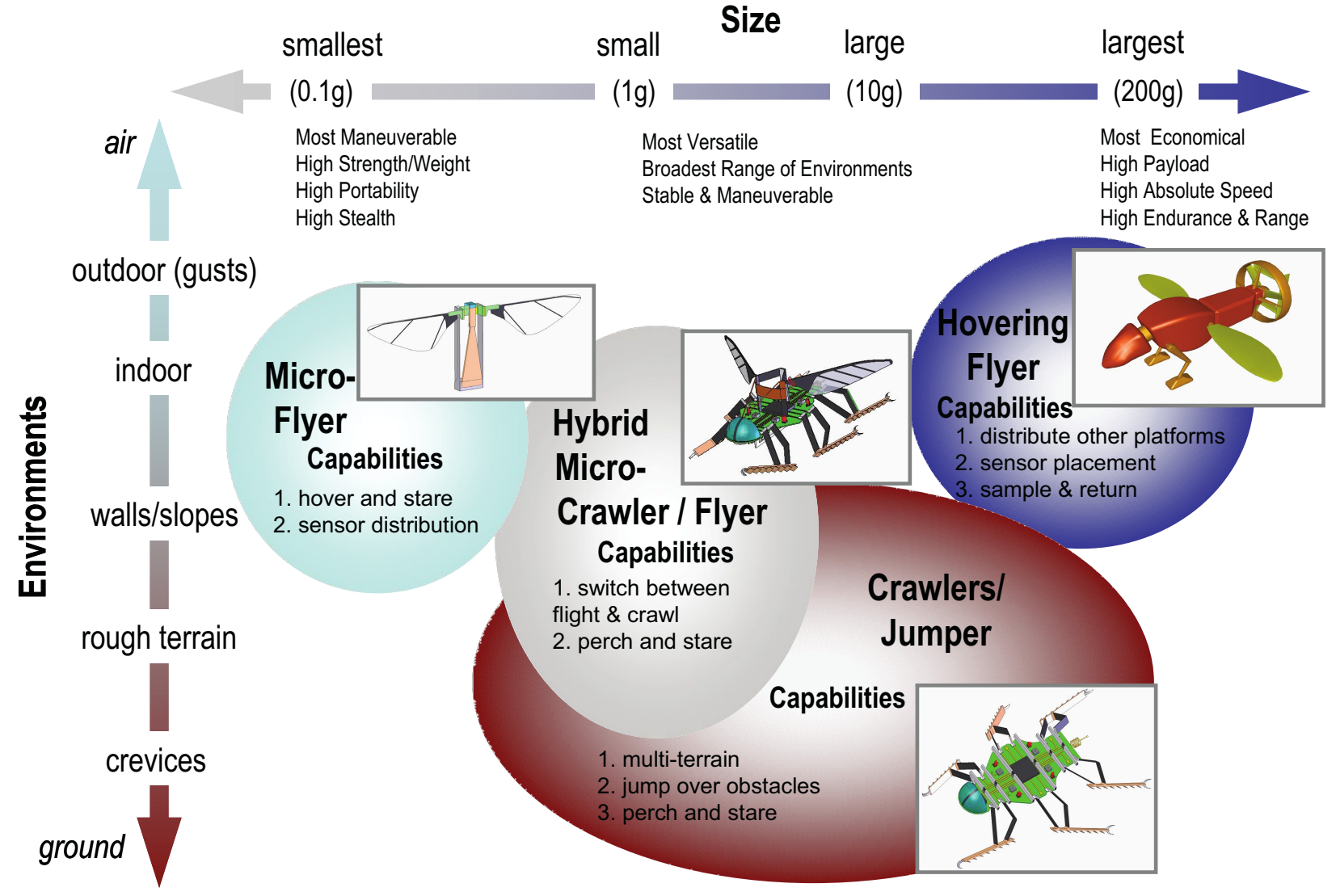

Figure 1. Envisioned platforms in the Micromechanics Center.

- Range and mission duration: the agents should be able to operate long enough to complete potentially complex missions. This presents particular challenges for smaller systems and flying agents, however there are also unique attributes to small-scale flight and ambulation that will be exploited to achieve maximally efficient locomotion.

All of these characteristics begin to form a concise description of the challenges presented to the Center on Microsystem Mechanics. Our objective is to create multiple scale aerial, ambulatory, and mixed-mode robotic agents that address each of these requirements (Figure 1). Since many natural species demonstrate each of the characteristics listed above, biological inspiration ${ }^{1,2}$ will be used in concert with engineering efforts.

\section{SCENARIOS}

The mission goals are to utilize a large number of microsystems to: enter and explore a building interior to find potential threats (Scenario 1), enter and explore caves or demolished buildings for potential threats (Scenario 2), and automatic spatial coverage of a wide area for perimeter defense (Scenario 3). In Scenario 1, a relatively benign environment (smooth floors, regular walls, quiescent air flow) is assumed, along with human mission control. Scenario 2 will require the ability to negotiate rough ground and handle unpredictable air flow. Scenario 3 , the most challenging, will require all these capabilities in addition to harsh environmental conditions such as high winds, rain, and mud. Our Center on Microsystem Mechanics will do the fundamental research to enable flying and ambulating platforms to have the needed mobility for these scenarios. 


\section{Scenario 1}

Small unit building search assuming smooth surfaces, quiescent air flow.

Warfighter(s) infiltrate a building by simultaneously deploying flyers through multiple openings. If a door is closed and the room is clear, the warfighter will need to enter and open the next door. If the room opens into a large space or hallways, more flyers will be deployed. Crawlers, which are simultaneously deployed by the flyer, climb to good perch locations to maintain line-of-sight communications and to assess confined spaces in the immediate vicinity. The smallest flying/crawling hybrids look for openings such as ventilation ducts, gaps under doors, or hiding spaces, and provide an ad hoc navigation network for the larger flyers. All microsystems continuously relay sensory data and assessments through the mobile network back to portable, light, and flexible readout displays for human operator assessment and control. Once critical targets are located, climbers will be deployed and crawlers

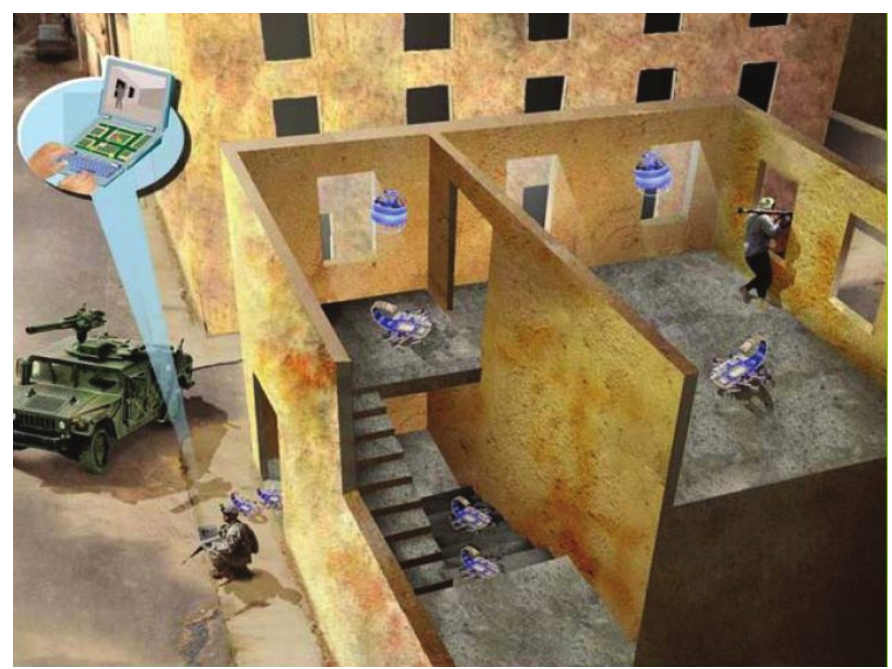

Figure 2. Small unit building search will be directed to human or autonomously specified locations for comprehensive sensor data collection, including visual, audio, IR/thermal, and olfactory data. If sampling and return is required, crawlers or hybrids will perform the action. Using low-power technology and energy harvesting, perched crawler assets can perform surveillance functions for weeks or months. Large flyers can be recovered for later use and refueling. Other smaller assets are designed to be disposable.

\section{Scenario 2}

Small unit cave search or demolished building assuming complex terrain (large obstacles, unstable surfaces, mud/water), unpredictable air flow (wind gusts), and undesirable visual conditions (low light, atmospheric obscurants).

Warfighter(s) gain entry access to demolished building or cave and deploy gust-tolerant flyers to give an initial assessment and map of the immediate environment, identifying closed spaces, IR signatures, video, and audio. Crawlers are simultaneously deployed to maintain line-of-sight connections and to assess confined spaces and difficult obstacles in the immediate vicinity. Combinations of hopping and gust tolerant hybrids are deployed to navigate difficult terrain and for immediate assessment of confined spaces that are deep in the building or cave. All microsystems continuously relay sensory data and assessments to portable, light, and flexible readout displays for human operator assessment and control. Once critical targets are located, climbers will be deployed and crawlers will be directed to hu-

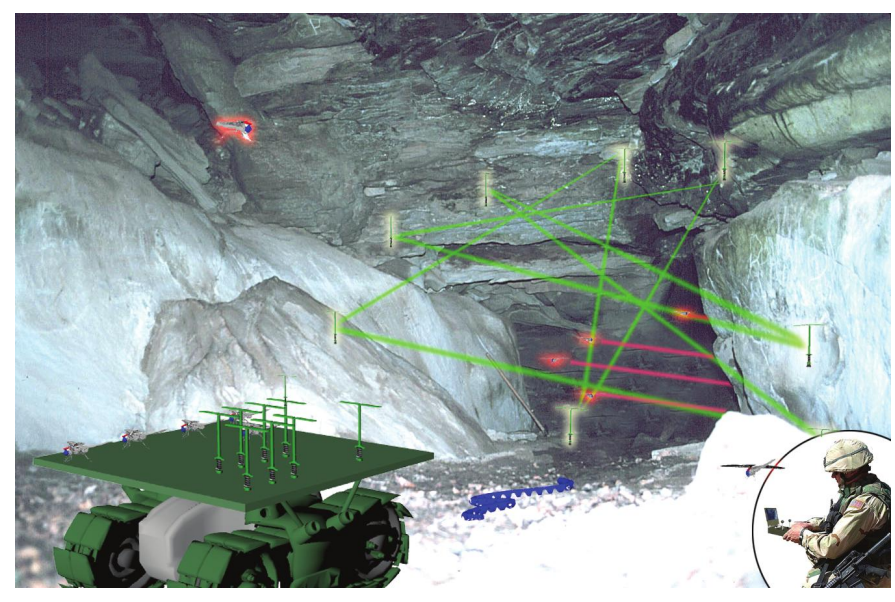

Figure 3. Cave search with complex terrain man or autonomously specified locations for comprehensive sensor data collection, including visual, audio, IR/thermal, and olfactory data. If sampling and return is required, crawlers or hybrids will perform the action. Once operations are complete, human operator will issue a recall command to reclaim high value assets. 


\section{Scenario 3}

Small unit perimeter defense assuming complete autonomy, complex terrain (large obstacles, unstable surfaces, mud/water), unpredictable air flow (wind gusts), and undesirable visual conditions (low light, atmospheric obscurants).

Warfighter(s) have secured a small section of an urban area including abandoned and demolished buildings. To effectively protect the area, every window, roof, and door must be covered by a persistent sensor network. For rapid deployment, hover capable flyers will fly near critical coverage points and launch micro crawlers onto building exteriors. The micro crawlers will adhere to masonry, metal, or glass walls, and deploy over distances of several meters to reach best locations for communication, observation, and protection from the elements. The network now monitors for any change in conditions, and if a threat is perceived (for example by a sensitive microphone detector), a large flyer with greater sensing capability, such as an IR viewer will be rapidly dispatched to investigate.

\section{CHALLENGES}

\subsection{Aeromechanics}

Research over the last 10 years has provided critical insight into the basis of low Reynolds number rotary and flapping wing aerodynamics, ${ }^{3,4}$ however a deep understanding of both the underlying physical mechanisms and useful engineering principles is still lacking. Thus, whereas scientists can now provide a physical explanation for how bees fly, they cannot present a set of engineering principles for building a bee-sized flying robot. Many envisioned flying devices push the realm of mechanical-flying devices into the size range of birds and insects; thus it is essential to formalize our understanding of aerodynamic mechanisms in this challenging and poorly mapped domain. Our strategy is to use a combination of multiple experimental and theoretical techniques to study the complex flows of low and intermediate Reynolds numbers, while continuing to gain insight from real animals that represent fully-functional examples of small-scale flying devices. The goal is to extract and formalize a set of principles that can be used in the construction of novel MAVs with expanded performance.
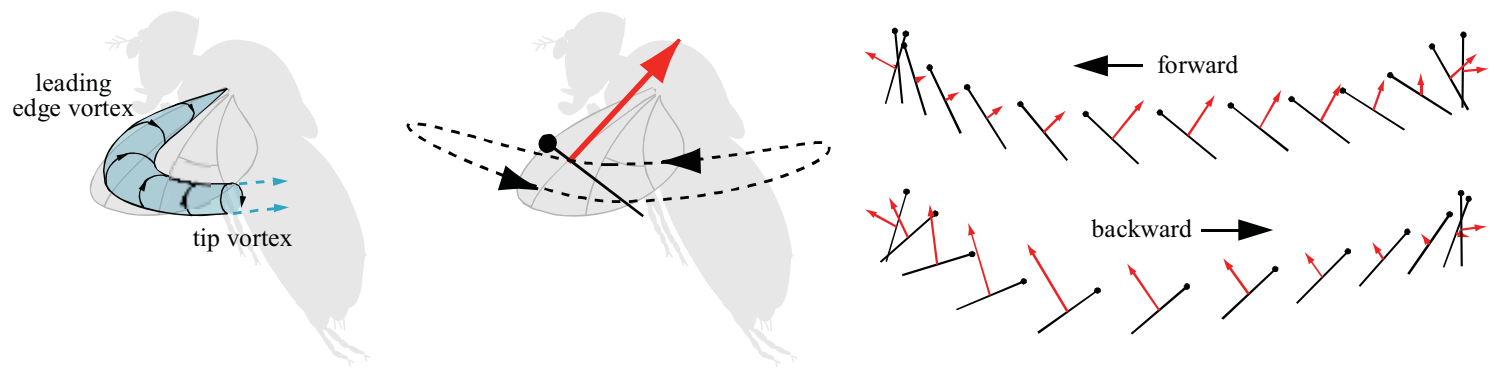

Figure 4. Flapping insect wings create a complex but stable leading edge vortex which enhances lift.

An example of one of the challenges concerns the nearly ubiquitous mechanics by which insect wings create the high lift forces necessary for hovering flight. Insects flap their wings back and forth at high angles of attack to create a separated leading edge vortex. While it is clear that insects take advantage of this unsteady, 3D, vortical flow, it is not clear how to make use of such a complex flow structure on a small wing, or under which conditions the insect strategy is more efficient than conventional mechanisms. ${ }^{5}$ The problem is that the complex flow structures created by flapping wings are sensitive to a host of interrelated parameters such as Reynolds number, reduced frequency, turbulence spectrums, Richardsons number, and Rossby number. Our lack of understanding of these effects and their interdependencies is a major obstacle in the design of bio-inspired MAVs. Even the miniaturization of conventional platforms (rather than bio-inspired designs) will benefit from this effort. For example, current hovering efficiency of existing micro-rotorcraft is roughly $65 \%$, compared to $80 \%$ or more for their full sized counterparts. ${ }^{6}$ The factors affecting this lower performance include the viscous dominated nature of flow at low Reynolds numbers, a critical topic in our investigation of flapping-based aerodynamics of birds and insects. 


\subsection{Ambulation}

Understanding dynamic ambulation of small mobile platforms on complex terrain, ${ }^{7,8}$ such as envisioned in the MAST scenarios, is an open problem for both scientific understanding and engineering design. Legs and feet must generate appropriate traction and adhesion forces on substrates which range from solid-like to liquid-like behavior at slopes ranging from 0 to 360 degrees. Animals use a wide variety of attachment and contact

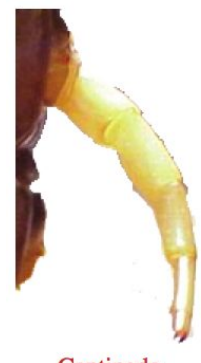

Centipede

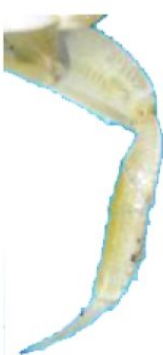

Crab

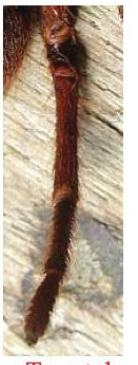

Tarantula

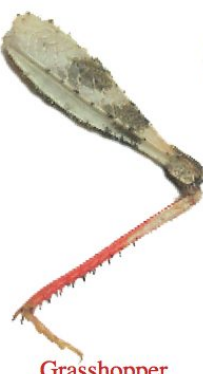

Grasshopper

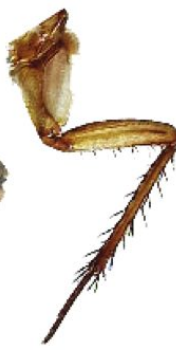

Cockroach

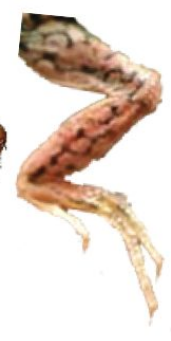

Lizard

Figure 5. Diverse legs designs that will provide inspiration for novel robot designs based on morphology and material properties and hairs to interact with surfaces, where the generated forces are complex functions of morphology, compliance, trajectory, topography, and surface mechanics. Leg stroke frequencies and disturbance response rates are too fast for sensory controlled foot placement, hence passive distributed mechanical feedback strategies are required. Understanding energy management is critical for both stabilization as well as long duration missions.

\subsection{Hybrid Aeromechanics and Ambulation}

The Center for Micromechanics will develop the fundamental principles of aeromechanics and ambulation to enable microsystem platforms to take off, fly, land, or run capably. The mission scenarios we consider require both range (for long distance exploration) and maneuverability (for exploration in confined indoor spaces). The research challenge is understanding the tradeoffs between locomotion modes in terms of robustness to disturbances, payload capacity, and efficiency. A hybrid approach to microsystem platform design, with a platform capable of both hover (through flapping) and high speed (fixed wing) flight, ${ }^{9}$ and a hybrid crawler/flyer capable of ambulation and flapping flight. Particular challenges are modelling aeroelastic coupling of flapping wings, and analogous terramechanical models for compliant limbs interacting with possibly flowing media. The overall goal is to develop a

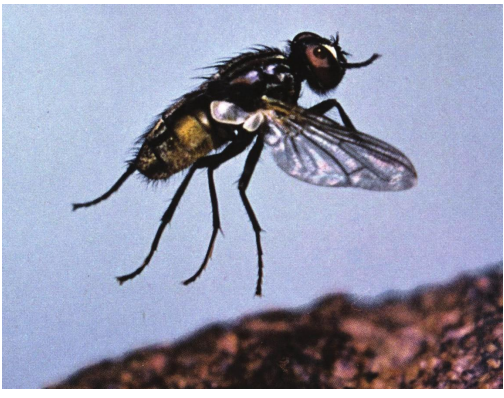

Figure 6. Flight initiation framework for a versatile platform which can adaptively choose the best locomotion mode to complete a mission.

\subsection{Multifunctional Actuation and Propulsion}

100 gram and smaller crawling, jumping, running, flying and hovering vehicles present extreme challenges in actuation, structures, design, and propulsion. ${ }^{10}$ Miniature actuation needs to not only efficiently provide high power to wings and legs, but to provide controllable impedance for steering and stabilization. The envisioned vehicles cannot be manufactured using existing materials and fabrication techniques: the size and weight constraints are too demanding and will require novel, integrated structures and actuators. Current battery technology has limited energy and power density for sustained hovering flight. Particularly for these small scales, management of kinetic and potential energy, controlled distribution of mechanical power, and optimal actuator partitioning is required for efficiency and stability.

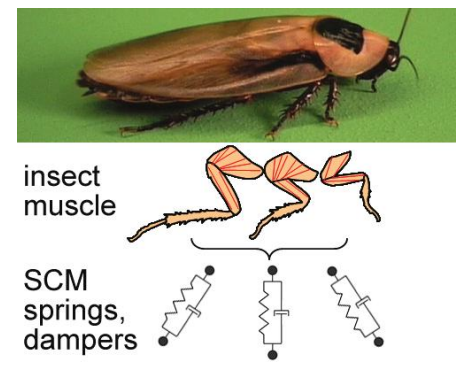

Figure 7. Mechanical energy storage and dissipation in legs 


\section{MICROSYSTEM MECHANICS CENTER PARTNERS}

The Center on Microsystem Mechanics is coordinated through the Alfred Gessow Rotorcraft Center (AGRC) at the University of Maryland, which serves as the Principal Member. The three General Members are the University of California at Berkeley, California Institute of Technology, and North Carolina A\&T State University. In addition the Center is comprised of two Supporting Member Universities, Harvard University and the Georgia Institute of Technology, and two Supporting Member small businesses, Centeye and Daedalus Flight Systems. This unique teaming relationship between biologists and engineers brings together experts in the areas of aeromechanics, ambulation, actuation, and propulsion.

The University of Maryland has extensive background in hovering MAVs. Research areas include design concepts, aeromechanics, insect-based sensing and control, morphing wings, micro-actuation, and micro-propulsion. UC Berkeley has extensive background in bio-inspired robotics, particularly flapping-wing actuation at the housefly scale, as well as ambulatory body and appendage design. Their work in micro-assembly and rapid prototyping has led to a new fabrication paradigm for millirobots. Caltech will focus on the aerodynamics and mechanics of flying animals for inspiration in the design of the MAVs. North Carolina A\&T State University has developed a unique electrospinning fabrication facility that is extensively used for the development of MAV components, including flapping wings. The Harvard Microrobotics Lab will focus on microrobots based upon Dipteran insects, and contains the worlds leading facilities for the rapid creation of the rigid, articulated, and actuated microstructures necessary for agile mobility of devices ranging from sub-millimeter to centimeter. Georgia Tech will focus on locomotion of terrestrial organisms and robots on flowing materials like granular media to develop interaction models for robots and organisms with the ground.

\section{ACKNOWLEDGMENTS}

The authors gratefully acknowledge support from the Army Research Laboratory under grant W911NF-08-20004 .

\section{REFERENCES}

[1] Full, R. J., "Dusing biological inspiration to build artificial life that locomotes," in [Evolutionary Robotics From Intelligent Robotics to Artificial Life, International Symposium, Tokyo, Japan], Gomi, T., ed., 110120, Springer-Verlag, Berlin (2001).

[2] Ritzman, R. E., Quinn, R. D., Watson, J. T., and Zill, S. N., "Insect walking and biorobotics: a relationship with mutual benefits," Bioscience 50, 23-33 (2000).

[3] Fry, S. N., Sayaman, R., and Dickinson, M. H., "The aerodynamics of free-flight maneuvers in drosophila," Science 300, 495-498 (2003).

[4] Dickinson, M. H., Lehmann, F. O., and Sane, S. P., "Wing rotation and the aerodynamic basis of insect flight," Science $\mathbf{2 8 4}$ (1999).

[5] Ramasamy, M. and Leishman, J. G., "Phase-locked piv measurements in the flow field of a flapping wing mav," Journal of Aircraft 43, 1876-1875 (2006).

[6] Hein, B. and Chopra, I., "Hover performance of a micro air vehicle: Rotor at low reynolds number," AHS International Specialists Meeting on Unmanned Rotorcraft: Design, Control and Testing, Phoenix, AZ (2005).

[7] Spagna, J. P., Goldman, D., Lin, P. C., Koditschek, D., and Full, R., "Distributed mechanical feedback in arthropods and robots simplifies control of rapid running on challenging terrain," Bioinspiration and Biomimetics 2, 9- (2007).

[8] Korff, W. L. and Goldman, D., "Escape at the solid/fluid interface: Impulsive accelerations of lizards on sand," Integrative and Comparative Biology 44, 585-(2004).

[9] Tarascio, M. J. and Chopra, I., "Design and development of a thrust augmented entomopter: An advaned flapping wing micro hovering air vehicle," Proceedings of 59th Annual Forum of the American Helicopter Society, Phoenix, AZ (2003).

[10] R.J. Wood, S. Avadhanula, R. S. and Fearing, R., "Microrobot design using fiber reinforced composites," J. of Mechanical Design 130 (2008). 